\title{
SOLVABILITY OF A NONLINEAR SECOND ORDER CONJUGATE EIGENVALUE PROBLEM ON A TIME SCALE
}

\author{
JOHN M. DAVIS, JOHNNY HENDERSON, K. RAJENDRA PRASAD, \\ AND WILLIAM YIN
}

Received 2 February 2000

We consider the nonlinear second order conjugate eigenvalue problem on a time scale: $y^{\Delta \Delta}(t)+\lambda a(t) f(y(\sigma(t)))=0, t \in[0,1], y(0)=0=y(\sigma(1))$. Values of the parameter $\lambda$ (eigenvalues) are determined for which this problem has a positive solution. The methods used here extend recent results by allowing for a broader class of functions for $a(t)$.

\section{Introduction}

In this paper, we are concerned with determining intervals of eigenvalues for boundary value problems for certain second order nonlinear differential equations on a time scale (also referred to, in the literature, as a measure chain). Much recent attention has been given to differential equations on time scales, and we refer the reader to $[2,4,8]$ for some historical works as well as to the more recent papers [1, 5, 6] and the book [10] for excellent references on these types of equations. Before introducing the problems of interest for this paper, we present some definitions and notation which are common to the recent literature. Our sources for this background material are the two papers by Erbe and Peterson $[5,6]$.

Definition 1.1. Let $\mathbb{T}$ be a closed subset of $\mathbb{R}$, and let $\mathbb{T}$ have the subspace topology inherited from the Euclidean topology on $\mathbb{R}$. The set $\mathbb{T}$ is referred to as a time scale or a measure chain. For $t<\sup \mathbb{T}$ and $r>\inf \mathbb{T}$, define the forward jump operator, $\sigma$, and the backward jump operator, $\rho$, respectively, by

$$
\sigma(t)=\inf \{\tau \in \mathbb{T} \mid \tau>t\} \in \mathbb{T}, \quad \rho(r)=\sup \{\tau \in \mathbb{T} \mid \tau<r\} \in \mathbb{T}, \quad \forall t, r \in \mathbb{T}
$$

If $\sigma(t)>t, t$ is said to be right scattered, and if $\rho(r)<r, r$ is said to be left scattered. If $\sigma(t)=t, t$ is said to be right dense, and if $\rho(r)=r, r$ is said to be left dense.

Remark 1.2. Here we provide some examples of time scales.

Copyright $(0) 2000$ Hindawi Publishing Corporation

Abstract and Applied Analysis 5:2 (2000) 91-99

2000 Mathematics Subject Classification: 34B15, 34G99, 39A12, 39A99

URL: http://aaa.hindawi.com/volume-5/S108533750000018X.html 
92 Solvability of a nonlinear second order conjugate eigenvalue problem ...

(1) $\mathbb{T}=\mathbb{R}$

(2) $\mathbb{T}=\mathbb{Z}$.

(3) $\mathbb{T}=\cup_{n=0}^{\infty}[2 n, 2 n+1]$.

(4) $\mathbb{T}=\{0\} \cup\{1\} \cup[2,3] \cup\{4\} \cup\{5\}$.

We refer to (1) as the continuous case and (2) as the discrete case for obvious reasons. However, we are not limited to these "extremes." Examples (3) and (4) are viable time scales as well. Note that (1) is both left dense and right dense for each $t \in \mathbb{T}$ while (2) is left scattered and right scattered for each $t \in \mathbb{T}$. On the other hand, (3) is left dense except at the left endpoints of each interval and right dense except at the right endpoints of each interval. Finally, (4) is left scattered for each $t \notin(2,3]$ and right scattered for each $t \notin[2,3)$.

Definition 1.3. For $x: \mathbb{T} \rightarrow \mathbb{R}$ and $t \in \mathbb{T}$ (if $t=\sup \mathbb{T}$, assume $t$ is not left scattered), define the delta derivative of $x(t)$, denoted by $x^{\Delta}(t)$, to be the number (when it exists), with the property that, for any $\epsilon>0$, there is a neighborhood, $U$, of $t$ such that

$$
\left|[x(\sigma(t))-x(s)]-x^{\Delta}(t)[\sigma(t)-s]\right| \leq \epsilon|\sigma(t)-s|, \quad \forall s \in U .
$$

The second delta derivative of $x(t)$ is defined by

$$
x^{\Delta \Delta}(t)=\left(x^{\Delta}\right)^{\Delta}(t)
$$

Remark 1.4. If $\mathbb{T}=\mathbb{R}$, then for $t \in \mathbb{R}$, and any differentiable $f: \mathbb{R} \rightarrow \mathbb{R}$, we have

$$
\sigma(t)=\rho(t)=t, \quad f^{\Delta}(t)=f^{\prime}(t) .
$$

Hence, differential equations on this time scale are ordinary differential equations.

On the other hand, if $\mathbb{T}=\mathbb{Z}$, then for $t \in \mathbb{Z}$ and any sequence $f: \mathbb{Z} \rightarrow \mathbb{R}$, we have

$$
\sigma(t)=t+1, \quad \rho(t)=t-1, \quad f^{\Delta}(t)=\Delta f(t) .
$$

Here $\Delta f(t)=f(t+1)-f(t)$ is the usual forward difference operator, and hence differential equations on this time scale are finite difference equations.

Definition 1.5. If $F^{\Delta}(t)=h(t)$, then define the integral by

$$
\int_{a}^{t} h(s) \Delta s=F(t)-F(a) .
$$

Remark 1.6. If $\mathbb{T}=\mathbb{R}$, then the integral is the usual Riemann integral. If $\mathbb{T}=\mathbb{Z}$, then the integral on this time scale is the usual summation operator.

Definition 1.7. Define the closed interval, $[a, b] \subset \mathbb{T}$ by

$$
[a, b]:=\{t \in \mathbb{T} \mid a \leq t \leq b\} .
$$

Other closed, open, half-open, and half-closed intervals in $\mathbb{T}$ are similarly defined. 
We want to consider the nonlinear conjugate eigenvalue problem

$$
\begin{gathered}
y^{\Delta \Delta}(t)+\lambda a(t) f(y(\sigma(t)))=0, \quad t \in[0,1], \\
y(0)=0=y(\sigma(1)) .
\end{gathered}
$$

We make the following assumptions throughout:

(A1) $a(t)$ is a nonnegative, continuous function defined on $(0, \sigma(1))$ such that

$$
0<\int_{0}^{\sigma(1)} h(s) a(s) \Delta s<\infty
$$

where $h(s)=\min \{\sigma(s), \sigma(1)-\sigma(s)\}$.

(A2) $f:[0, \infty) \rightarrow[0, \infty)$ is continuous and

$$
f_{0}=\lim _{x \rightarrow 0^{+}} \frac{f(x)}{x}, \quad f_{\infty}=\lim _{x \rightarrow \infty} \frac{f(x)}{x},
$$

both exist.

We remark that by a solution of (1.8) and (1.9), we mean a function $u:\left[0, \sigma^{2}(1)\right] \rightarrow$ $\mathbb{R}$, where $u$ satisfies $(1.8)$ on $[0,1]$ and the boundary conditions (1.9). We further remark that, if $u$ is a nonnegative solution of (1.8) and (1.9), then $u^{\Delta \Delta}(t) \leq 0$ on [0,1], and we will say $u$ is concave on $\left[0, \sigma^{2}(1)\right]$.

We note that (A1) allows for $a(t) \equiv 0$ on some subinterval(s) of $[0, \sigma(1)]$ and allows $a(t)$ to have a singularity at $t=0$ and/or $t=1$. Jiang and Liu [9] provided

$$
a(t)=t^{-\alpha}(1-t)^{-\beta}(|\cos 2 \pi t|+\cos 2 \pi t)
$$

as an example. Assumption (A1) is important because it admits a larger class of functions than those allowed in [3].

This paper constitutes an extension of the recent work by Erbe and Peterson [6] and Chyan and Henderson [3] in which they obtained positive solutions of (1.8) and (1.9) for all $0<\lambda<\infty$ assuming that $f$ is either superlinear or sublinear. The solutions obtained in $[3,6]$ were found to belong to the intersection of a cone with an annular type region.

Now we state a Green's function inequality which is fundamental in the proof of our main result.

THEOREM 1.8. Let $G(t, s)$ denote the Green's function for the homogeneous problem

$$
-y^{\Delta \Delta}=0
$$

satisfying the boundary conditions (1.9). Define

$$
g(t)=\min \{t, \sigma(1)-t\}, \quad h(s)=\min \{\sigma(s), \sigma(1)-\sigma(s)\} .
$$

Then

$$
\frac{1}{\sigma(1)} g(t) h(s) \leq G(t, s) \leq \frac{\sigma(s)(\sigma(1)-\sigma(s))}{\sigma(1)}, \quad(t, s) \in[0, \sigma(1)] \times[0,1] .
$$


94 Solvability of a nonlinear second order conjugate eigenvalue problem ...

Proof. Erbe and Peterson [6] have found the explicit form of the Green's function to be

$$
G(t, s)= \begin{cases}\frac{t(\sigma(1)-\sigma(s))}{\sigma(1)}, & t \in[0, s], \\ \frac{\sigma(s)(\sigma(1)-t)}{\sigma(1)}, & t \in[\sigma(s), \sigma(1)] .\end{cases}
$$

From the above inequality, we can see for $t \in[0, s]$,

$$
G(t, s)=\frac{t(\sigma(1)-\sigma(s))}{\sigma(1)} \leq \frac{s(\sigma(1)-\sigma(s))}{\sigma(1)} \leq \frac{\sigma(s)(\sigma(1)-\sigma(s))}{\sigma(1)} .
$$

For $t \in[\sigma(s), \sigma(1)]$,

$$
G(t, s)=\frac{\sigma(s)(\sigma(1)-t)}{\sigma(1)} \leq \frac{\sigma(s)(\sigma(1)-\sigma(s))}{\sigma(1)} .
$$

Finally, if $(t, s) \in[0, \sigma(1)] \times[0,1]$, then

$$
G(t, s) \geq g(t) h(s) \frac{1}{\sigma(1)},
$$

where $g(t)=\min \{t, \sigma(1)-t\}$ and $h(s)=\min \{\sigma(s), \sigma(1)-\sigma(s)\}$. (We remark that in the case when $s$ is right dense, $h(s)=g(s)$.)

Theorem 1.9 (Guo and Lakshmikantham [7, page 94]). Let E be a Banach space, $K \subseteq E$ be a cone, and suppose that $\Omega_{1}, \Omega_{2}$ are open subsets of $E$ with $0 \in \Omega_{1}$ and $\bar{\Omega}_{1} \subset \Omega_{2}$. Suppose further that $A: K \cap\left(\bar{\Omega}_{2} \backslash \Omega_{1}\right) \rightarrow K$ is a completely continuous operator such that either

(i) $\|A u\| \leq\|u\|, u \in K \cap \partial \Omega_{1}$ and $\|A u\| \geq\|u\|, u \in K \cap \partial \Omega_{2}$, or

(ii) $\|A u\| \geq\|u\|, u \in K \cap \partial \Omega_{1}$ and $\|A u\| \leq\|u\|, u \in K \cap \partial \Omega_{2}$

holds. Then $A$ has a fixed point in $K \cap\left(\bar{\Omega}_{2} \backslash \Omega_{1}\right)$.

\section{Main results}

In this section, we apply Theorem 1.9 to the eigenvalue problem (1.8) and (1.9). Throughout this section, we assume $\sigma(1)$ is right dense so that $G(t, s) \geq 0$, for $t \in[0, \sigma(1)], s \in[0, \sigma(1)]$.

Take our Banach space to be $\mathscr{B}=\left\{x:\left[0, \sigma^{2}(1)\right] \rightarrow \mathbb{R} \mid x\right.$ is continuous $\}$ with the norm

$$
\|x\|=\sup _{t \in\left[0, \sigma^{2}(1)\right]}|x(t)|
$$

Define the cone $\mathscr{P} \subset \mathscr{B}$ by

$$
\mathscr{P}=\left\{x \in \mathscr{B} \mid x(t) \geq \frac{1}{\sigma(1)} g(t)\|x\|, t \in\left[0, \sigma^{2}(1)\right]\right\} .
$$


Let $\delta, \omega \in(0, \sigma(1))$ and $[\delta, \omega] \subset[0, \sigma(1)]$ be chosen such that

$$
\int_{\delta}^{\sigma(\omega)} a(s) h(s) d s>0,
$$

and choose $\tau \in[\delta, \sigma(\omega)]$ such that

$$
g(\tau)=\max _{t \in[\delta, \sigma(\omega)]} g(t) .
$$

We are now ready to state our first theorem which establishes an open interval of values of $\lambda$ for which (1.8) and (1.9) has a positive solution.

Theorem 2.1. Suppose that (A1) and (A2) hold and that $\sigma(1)$ is right dense. Then for each $\lambda$ satisfying

$$
\frac{(\sigma(1))^{2}}{g(\tau) \delta \int_{\delta}^{\sigma(\omega)} h(s) a(s) \Delta s f_{\infty}}<\lambda<\frac{\sigma(1)}{\int_{0}^{\sigma(1)} \sigma(s)(\sigma(1)-\sigma(s)) a(s) \Delta s f_{0}},
$$

there exists at least one solution of (1.8) and (1.9) in $\mathscr{P}$.

Proof. Let $\lambda$ be given as in (2.5) and let $\varepsilon>0$ be such that

$$
\frac{(\sigma(1))^{2}}{g(\tau) \delta \int_{\delta}^{\sigma(\omega)} h(s) a(s) \Delta s\left(f_{\infty}-\epsilon\right)} \leq \lambda \leq \frac{\sigma(1)}{\int_{0}^{\sigma(1)} \sigma(s)(\sigma(1)-\sigma(s)) a(s) \Delta s\left(f_{0}+\epsilon\right)}
$$

We note that $y(t)$ is a solution of (1.8) and (1.9) if and only if

$$
y(t)=\lambda \int_{0}^{\sigma(1)} G(t, s) a(s) f(y(\sigma(s))) \Delta s, \quad t \in[0, \sigma(1)] .
$$

Motivated by this, we define the operator $T: \mathscr{P} \rightarrow \mathscr{B}$ by

$$
(T y)(t)=\lambda \int_{0}^{\sigma(1)} G(t, s) a(s) f(y(\sigma(s))) \Delta s, \quad y \in \mathscr{P} .
$$

We seek a fixed point of $T$ in $\mathscr{P}$. We prove this by showing that the conditions in Theorem 1.9 hold.

First, if $y \in \mathscr{P}$ then

$$
\begin{aligned}
(T y)(t) & =\lambda \int_{0}^{\sigma(1)} G(t, s) a(s) f(y(\sigma(s))) \Delta s \\
& \leq \lambda \int_{0}^{\sigma(1)} \frac{1}{\sigma(1)} \sigma(s)(\sigma(1)-\sigma(s)) a(s) f(y(\sigma(s))) \Delta s,
\end{aligned}
$$

and so

$$
\|T y\| \leq \lambda \int_{0}^{\sigma(1)} \frac{1}{\sigma(1)} \sigma(s)(\sigma(1)-\sigma(s)) a(s) f(y(\sigma(s))) \Delta s .
$$


96 Solvability of a nonlinear second order conjugate eigenvalue problem ...

Next, if $y \in \mathscr{P}$, then by (1.15) and inequality (2.10) we see that

$$
\begin{aligned}
(T y)(t) & =\lambda \int_{0}^{\sigma(1)} G(t, s) a(s) f(y(\sigma(s))) \Delta s \\
& \geq \lambda \int_{0}^{\sigma(1)} \frac{1}{\sigma(1)} g(t) h(s) a(s) f(y(\sigma(s))) \Delta s \\
& \geq \lambda g(t) \int_{0}^{\sigma(1)} \frac{1}{\sigma(1)} \frac{\sigma(s)(\sigma(1)-\sigma(s))}{\sigma(1)} a(s) f(y(\sigma(s))) \Delta s \\
& \geq g(t) \frac{1}{\sigma(1)} \lambda \int_{0}^{\sigma(1)} \frac{1}{\sigma(1)} \sigma(s)(\sigma(1)-\sigma(s)) a(s) f(y(\sigma(s))) \Delta s \\
& \geq g(t) \frac{1}{\sigma(1)}\|T y\| .
\end{aligned}
$$

Hence $T: \mathscr{P} \rightarrow \mathscr{P}$. Standard arguments show that $T$ is completely continuous.

We begin with $f_{0}$. By the definition of $f_{0}$, there exists an $H_{1}>0$ such that $f(x) \leq$ $\left(f_{0}+\varepsilon\right) x$ for $0<x \leq H_{1}$. Let $\Omega_{1}=\left\{x \in \mathscr{B} \mid\|x\|<H_{1}\right\}$ and choose $y \in \mathscr{P}$ with $\|y\|=H_{1}$. Using (1.15) and the assumed right density of $\sigma(1)$, for $t \in\left[0, \sigma^{2}(1)\right]$,

$$
\begin{aligned}
(T y)(t) & =\lambda \int_{0}^{\sigma(1)} G(t, s) a(s) f(y(\sigma(s))) \Delta s \\
& \leq \lambda \int_{0}^{\sigma(1)} \frac{1}{\sigma(1)} \sigma(s)(\sigma(1)-\sigma(s)) a(s)\left(f_{0}+\varepsilon\right) y(\sigma(s)) \Delta s \\
& \leq \lambda \int_{0}^{\sigma(1)} \frac{1}{\sigma(1)} \sigma(s)(\sigma(1)-\sigma(s)) a(s) \Delta s\left(f_{0}+\varepsilon\right)\|y\| \\
& \leq \lambda \int_{0}^{\sigma(1)} h(s) a(s) \Delta s\left(f_{0}+\varepsilon\right)\|y\| \leq\|y\| .
\end{aligned}
$$

The last inequality follows from the right side of (2.6). Therefore, $\|T y\| \leq\|y\|$ and in particular

$$
\|T y\| \leq\|y\|, \quad y \in \mathscr{P} \cap \partial \Omega_{1} .
$$

Now we turn our attention to $f_{\infty}$. By the definition of $f_{\infty}$, there exists an $H_{2}>H_{1}$ such that $f(x) \geq\left(f_{\infty}-\varepsilon\right) x$ for $x \geq H_{2}$. If $y \in \mathscr{P}$ with $\|y\|=H_{2}$, if $y \in \mathscr{P}$ with $\|y\|=H_{2}$, then for $t \in[\delta, \omega]$, we have

$$
y(t) \geq \frac{1}{\sigma(1)} g(t)\|y\|=\frac{1}{\sigma(1)} g(t) H_{2} \geq \frac{1}{\sigma(1)} \delta H_{2} .
$$


Using (2.6) and (2.14), we get

$$
\begin{aligned}
(T y)(\tau) & =\lambda \int_{0}^{\sigma(1)} G(\tau, s) a(s) f(y(\sigma(s))) \Delta s \\
& \geq \lambda \int_{\delta}^{\sigma(\omega)} \frac{1}{\sigma(1)} g(\tau) h(s) a(s) f(y(\sigma(s))) \Delta s \\
& \geq \lambda \int_{\delta}^{\sigma(\omega)} g(\tau) h(s) a(s) y(\sigma(s)) \Delta s\left(f_{\infty}-\varepsilon\right) \\
& \geq \lambda g(\tau) \int_{\delta}^{\sigma(\omega)} \frac{1}{\sigma(1)} h(s) \frac{1}{\sigma(1)} \delta H_{2} a(s) \Delta s\left(f_{\infty}-\epsilon\right) \\
& =\lambda g(\tau) \frac{1}{(\sigma(1))^{2}} \delta H_{2} \int_{\delta}^{\sigma(\omega)} h(s) a(s) \Delta s\left(f_{\infty}-\epsilon\right) \\
& \geq H_{2}=\|y\| .
\end{aligned}
$$

If we define

$$
\Omega_{2}=\left\{x \in \mathscr{P} \mid\|x\|<H_{2}\right\},
$$

then we have shown that

$$
\|T y\| \geq\|y\|, \quad y \in \mathscr{P} \cap \partial \Omega_{2} .
$$

An application of Theorem 1.9 yields the conclusion of our theorem.

THEOREM 2.2. Suppose (A1) and (A2) hold. Then for each $\lambda$ satisfying

$$
\frac{(\sigma(1))^{2}}{g(\tau) \delta \int_{\delta}^{\sigma(\omega)} h(s) a(s) \Delta s f_{0}}<\lambda<\frac{\sigma(1)}{\int_{0}^{\sigma(1)} \sigma(s)(\sigma(1)-\sigma(s)) a(s) \Delta s f_{\infty}}
$$

there exists at least one solution of (1.8) and (1.9) in $\mathscr{P}$.

Proof. Let $\lambda$ be given as in (2.18) and let $\eta$ be given such that

$$
\begin{aligned}
& \frac{(\sigma(1))^{2}}{g(\tau) \delta \int_{\delta}^{\sigma(\omega)} h(s) a(s) \Delta s\left(f_{0}-\eta\right)} \\
& \quad \leq \lambda \leq \frac{\sigma(1)}{\int_{0}^{\sigma(1)} \sigma(s)(\sigma(1)-\sigma(s)) a(s) \Delta s\left(f_{\infty}+\eta\right)}
\end{aligned}
$$

Let $T$ be the cone preserving, completely continuous operator defined in (2.8).

Beginning with $f_{0}$, there exists an $H_{1}>0$ such that $f(x) \geq\left(f_{0}-\eta\right) x$ for $0<x \leq$ $H_{1}$. Choose $y \in \mathscr{P}$ with $\|y\|=H_{1}$. Note that for $t \in[\delta, \omega]$,

$$
y(t) \geq \frac{1}{\sigma(1)} g(t)\|y\|=\frac{1}{\sigma(1)} g(t) H_{1} \geq \frac{1}{\sigma(1)} \delta H_{1} .
$$


98 Solvability of a nonlinear second order conjugate eigenvalue problem ...

Using inequality (2.20), and the left side of (2.19), we obtain

$$
\begin{aligned}
(T y)(\tau) & =\lambda \int_{0}^{\sigma(1)} G(\tau, s) a(s) f(y(\sigma(s))) \Delta s \\
& \geq \lambda \int_{\delta}^{\sigma(\omega)} \frac{1}{\sigma(1)} g(\tau) h(s) a(s)\left(f_{0}-\varepsilon\right) y(\sigma(s)) \Delta s \\
& \geq \lambda \frac{1}{\sigma(1)} g(\tau) \frac{1}{\sigma(1)} \delta H_{1} \int_{\delta}^{\sigma(\omega)} h(s) a(s) \Delta s\left(f_{0}-\eta\right) \\
& \geq H_{1}=\|y\| .
\end{aligned}
$$

Thus, $\|T y\| \geq\|y\|$. So, if we define

$$
\Omega_{1}=\left\{x \in \mathscr{B}:\|x\|<H_{1}\right\},
$$

then we have shown that

$$
\|T y\| \geq\|y\|, \quad y \in \mathscr{P} \cap \partial \Omega_{1} .
$$

Now we turn our attention to $f_{\infty}$. By the definition of $f_{\infty}$, there exists $\bar{H}_{2}>H_{1}$ such that $f(x) \leq\left(f_{\infty}+\eta\right) x$ for $x \geq \bar{H}_{2}$. We have two cases: when $f$ is bounded and when $f$ is not bounded. First, suppose $f$ is bounded, then there exists $N>0$ such that $f(x) \leq N$ for all $0<x<\infty$. Let

$$
H_{2}=\max \left\{2 \bar{H}_{2}, N \lambda \int_{0}^{\sigma(1)} \frac{1}{\sigma(1)}(\sigma(1)-\sigma(s)) a(s) \Delta s\right\} .
$$

If $y \in \mathscr{P}$ with $\|y\|=H_{2}$, then we have

$$
\begin{aligned}
(T y)(t) & =\lambda \int_{0}^{\sigma(1)} G(t, s) a(s) f(y(\sigma(s))) \Delta s \\
& \leq N \lambda \int_{0}^{\sigma(1)} G(t, s) a(s) \Delta s \\
& \leq N \lambda \int_{0}^{\sigma(1)} \frac{1}{\sigma(1)} \sigma(s)(\sigma(1)-\sigma(s)) a(s) \Delta s \\
& \leq H_{2}=\|y\| .
\end{aligned}
$$

Now suppose $f$ is unbounded, let $H_{2}>\max \left\{2 H_{1}, \bar{H}_{2}\right\}$ be such that $f(x) \leq f\left(H_{2}\right)$ for $0<x \leq H_{2}$. If $y \in \mathscr{P}$ with $\|y\|=H_{2}$, then we have

$$
\begin{aligned}
(T y)(t) & =\lambda \int_{0}^{\sigma(1)} G(t, s) a(s) f(y(\sigma(s))) \Delta s \\
& \leq \lambda \int_{0}^{\sigma(1)} \frac{1}{\sigma(1)} \sigma(s)(\sigma(1)-\sigma(s)) a(s) f\left(H_{2}\right) \Delta s \\
& \leq \frac{1}{\sigma(1)} \lambda \int_{0}^{\sigma(1)} \sigma(s)(\sigma(1)-\sigma(s)) a(s) \Delta s\left(f_{\infty}+\eta\right) H_{2} \\
& \leq H_{2}=\|y\| .
\end{aligned}
$$


Thus, $\|T y\| \leq\|y\|$. So, if we define

$$
\Omega_{2}=\left\{x \in B \mid\|x\|<H_{2}\right\},
$$

then we have shown that

$$
\|T y\| \leq\|y\|, \quad y \in \mathscr{P} \cap \partial \Omega_{2} .
$$

An application of Theorem 1.9 yields the conclusion of our theorem.

\section{References}

[1] R. P. Agarwal and M. Bohner, Basic calculus on time scales and some of its applications, Results Math. 35 (1999), no. 1-2, 3-22. MR 99m:41030. Zbl 927.39003.

[2] B. Aulbach and S. Hilger, Linear dynamic processes with inhomogeneous time scale, Nonlinear Dynamics and Quantum Dynamical Systems (Gaussig, 1990), Math. Res., vol. 59, Akademie-Verlag, Berlin, 1990, pp. 9-20. MR 91g:58266. Zbl 719.34088.

[3] C. J. Chyan and J. Henderson, Eigenvalue problems for nonlinear differential equations on a measure chain, J. Math. Anal. Appl. 245 (2000), no. 2, 547-559. CMP 1758555. Zbl 953.34068.

[4] L. Erbe and S. Hilger, Sturmian theory on measure chains, Differential Equations Dynam. Systems 1 (1993), no. 3, 223-244. MR 95b:39002. Zbl 868.39007.

[5] L. Erbe and A. Peterson, Green's functions and comparison theorems for differential equations on measure chains, Dynam. Contin. Discrete Impuls. Systems 6 (1999), no. 1, 121-137. MR 2000a:34028. Zbl 938.34027.

[6] _ Positive solutions for a nonlinear differential equation on a measure chain, Math. Comput. Modelling 32 (2000), no. 5-6, 571-585. CMP 1791 165. Zbl 992.11479.

[7] D. J. Guo and V. Lakshmikantham, Nonlinear Problems in Abstract Cones, Notes and Reports in Mathematics in Science and Engineering, vol. 5, Academic Press, Massachusetts, 1988. MR 89k:47084. Zbl 661.47045.

[8] S. Hilger, Analysis on measure chains - a unified approach to continuous and discrete calculus, Results Math. 18 (1990), no. 1-2, 18-56. MR 91m:26027. Zbl 722.39001.

[9] D. Jiang and H. Liu, Existence of positive solutions to $(k, n-k)$ conjugate boundary value problems, Kyushu J. Math. 53 (1999), no. 1, 115-125. MR 99k:34045. Zbl 928.34020.

[10] V. Lakshmikantham, S. Sivasundaram, and B. Kaymakcalan, Dynamic Systems on Measure Chains, Mathematics and its Applications, vol. 370, Kluwer Academic Publishers Group, Dordrecht, 1996. MR 97j:34002. Zbl 869.34039.

John M. Davis: Department of Mathematics, Baylor University, Waco, TX 76798, USA E-mail address: John_M_Davis@baylor.edu

Johnny Henderson: Department of Mathematics, Auburn University, Auburn, AL 36849, USA

E-mail address: hendej2@mail.auburn.edu

K. Rajendra Prasad: Department of Applied Mathematics, Andhra University, VISAKHAPATNAM 530003, INDIA

E-mail address: rajendra92@hotmail.com

William Yin: Department of Mathematics, LaGrange College, LaGrange, GA 30240, USA

E-mail address: wyin@lgc.edu 


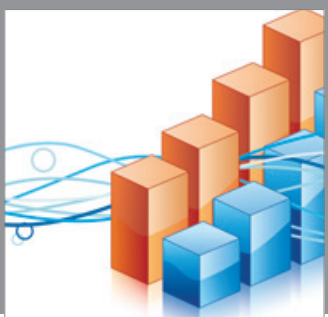

Advances in

Operations Research

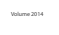

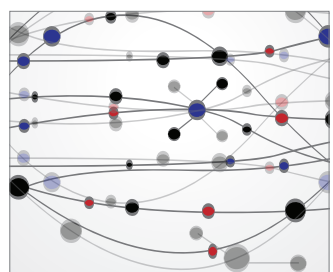

\section{The Scientific} World Journal
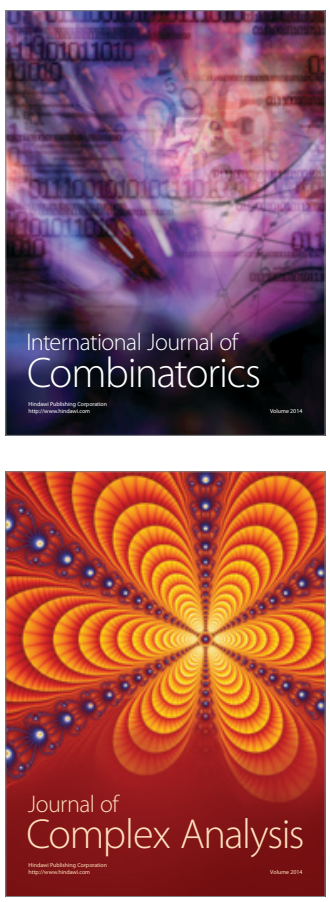

International Journal of

Mathematics and

Mathematical

Sciences
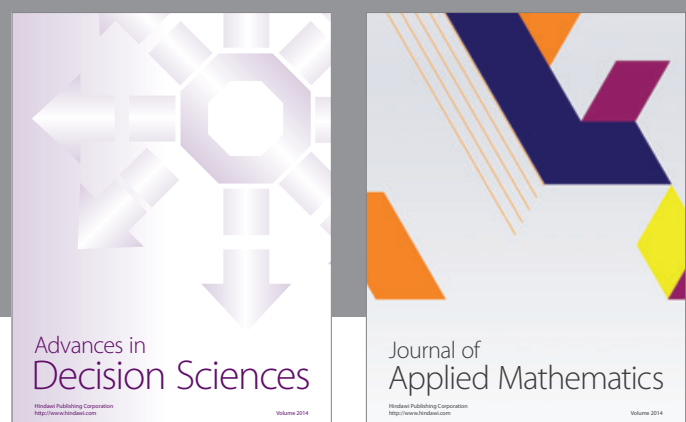

Journal of

Applied Mathematics
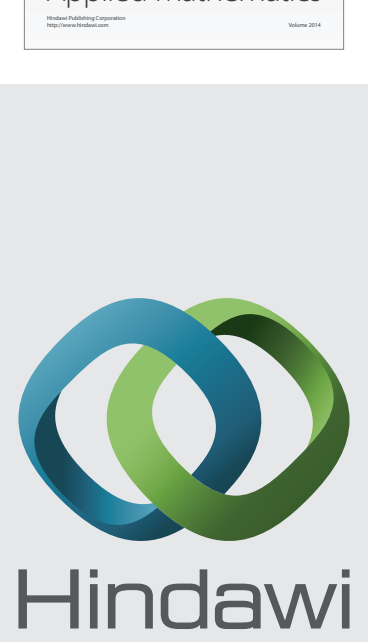

Submit your manuscripts at http://www.hindawi.com
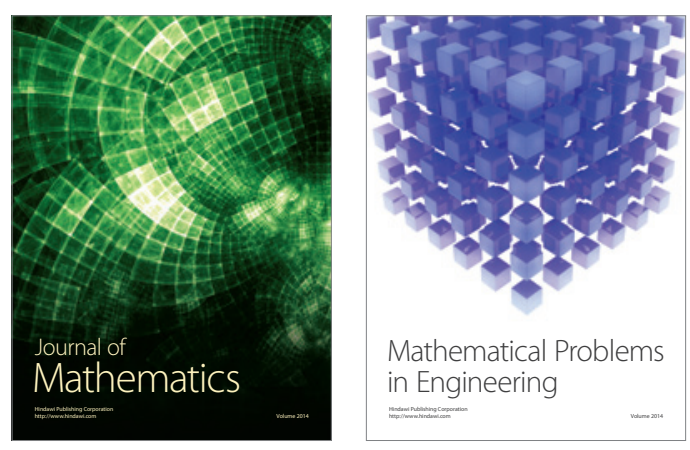

Mathematical Problems in Engineering
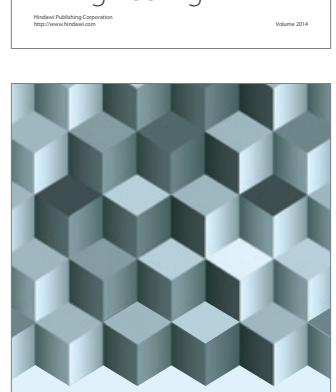

Journal of

Function Spaces
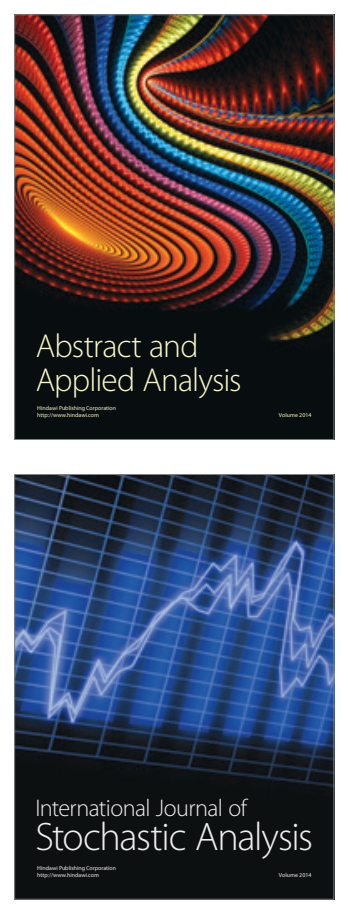

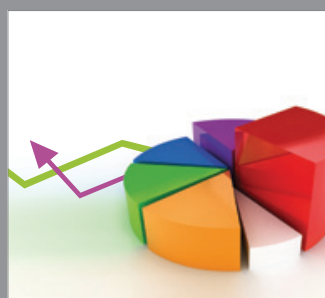

ournal of

Probability and Statistics

Promensencen
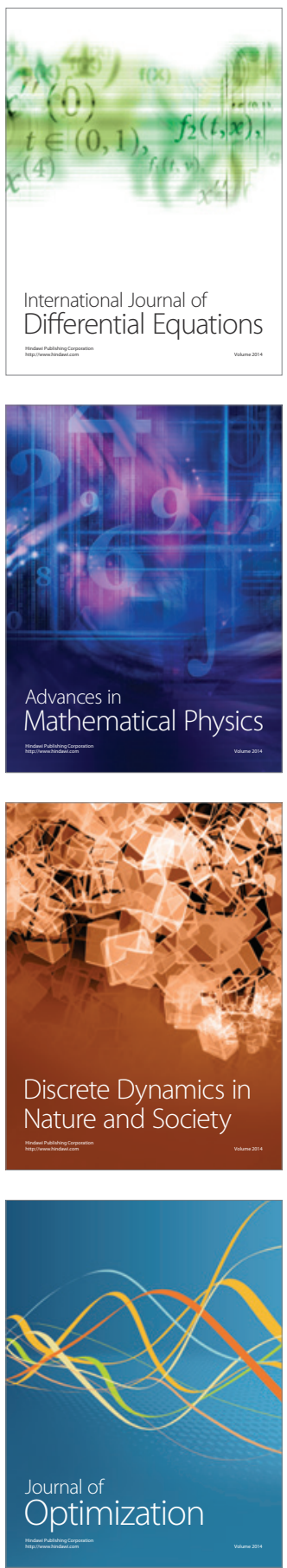